\title{
A Novel Case of Diabetic Muscle Necrosis in a Patient with Cystic Fibrosis-Related Diabetes
}

\author{
Sreelatha Chalasani, MD; Shankar S. Bettadahalli, MD; Satya V. Bhupathi, MD; and \\ Vijay H. Aswani, MD, PhD
}

\begin{abstract}
Cystic fibrosis is a recessive autosomal disease caused by mutations in the cystic fibrosis transmembrane conductance regulator gene. Cystic fibrosis-related diabetes (CFRD) is a common comorbidity of cystic fibrosis. Diabetic myonecrosis is a rare self-limited complication of poorly controlled diabetes mellitus that commonly presents with acute, intense pain and swelling of lower extremities and responds well to conservative management. We report the first case of diabetic myonecrosis in a patient with CFRD.
\end{abstract}

Keywords: Cystic Fibrosis; Complications; Physiopathology; Diabetic myonecrosis

Corresponding Author:

Vijay H. Aswani, MD, PhD

Marshfield Clinic

Department of Internal Medicine \& Pediatrics

1000 North Oak Avenue, Mail stop: IF3

Marshfield, WI 54449 USA

Tel: 7| $5-387-5600$

Fax: 7|5-389-3322

Email: aswani.vijay@marshfieldclinic.org

Received: September 25, 2012

Ist Revision: January 3, 2013

2nd Revision: February 15, 2013

Accepted: February 20, 2013

doi: $10.3121 / \mathrm{cmr} .2013 .1124$
O

ystic fibrosis (CF) is a recessive autosomal disease caused by mutations in the cystic fibrosis transmembrane conductance regulator (CFTR) gene. The CFTR protein is an epithelial ion channel contributing to the regulation of absorption and secretion of salt and water in various tissues including lung, sweat glands, pancreas, and the gastrointestinal tract. ${ }^{1}$ Cystic fibrosis-related diabetes (CFRD) is a common comorbidity of $\mathrm{CF}^{2}$ Diabetic myonecrosis is a rare, selflimited complication of poorly controlled diabetes mellitus (DM) that commonly presents with acute, intense pain and swelling of the lower extremities, with the thigh being the most commonly affected area, and responds well to conservative management. ${ }^{3}$

Notably, this case is unique in that it represents the first report of diabetic myonecrosis in an adult patient with CF. Adults with CF are a small but growing patient population due to increased survival. In 1991, over $32 \%$ of people in the United States Cystic Fibrosis Foundation Patient Registry were ages 18 years or older. This percentage increased to more than $48 \%$ in 2011 . The registry contained 27,111 patients with CF in 2011, of whom 13,094 were adults. ${ }^{4}$ The median age of survival of patients with $\mathrm{CF}$ is now into the late 30s. This presents providers of adult CF patients with new challenges in managing these patients. There is a need for additional information in diagnosing and treating these patients in the inpatient and outpatient settings where they may report with acute singularly unique presentations, as this case illustrates. Recognizing that CFRD can occur in adults with CF is important, because adults with CF have a tendency to develop CFrelated arthropathy, ${ }^{5}$ which is more likely to be suspected when these patients present with lower extremity pain. Diabetic myonecrosis is not currently included among differential diagnoses in patients with $\mathrm{CF}$, because it has never been reported in CFRD patients before, and yet it is important that this diagnosis not be missed. 


\section{Case Presentation}

A Caucasian man, age 32 years, with $\mathrm{CF}$, homozygous for delta F508 mutations, who underwent lung transplant in 2008, with uncontrolled CFRD presented with worsening pain in the left thigh and calf. The pain had been present for 3 weeks and was exacerbated by weight bearing. The patient attributed the origin of the pain to muscle strain secondary to increased physical activity. He reported no other complaints. He denied any recent trauma or any other constitutional symptoms including fever and chills.

Medical history included a diagnosis of $\mathrm{CF}$ with double lung transplant 4 years earlier. He also suffered from CFRD, with diabetic nephropathy as evidenced by class III chronic kidney disease and urine microalbuminuria, diabetic retinopathy seen on dilated eye exam, and peripheral neuropathy as evidenced by symptoms and decreased foot sensation. He also presented with hyperparathyroidism secondary to chronic kidney disease, hypertension, had a history of an ileostomy for small bowel obstruction, and poorly controlled DM of 18 years' duration. Subsequent to his lung transplant, he was maintained on ongoing immunosuppressive therapy (with mycophenolate and tacrolimus) and steroid therapy (prednisone $5 \mathrm{mg}$ by mouth daily), which exacerbated his CFRD. His other medications included amlodipine, metoprolol, insulin glargine, insulin lispro and lipaseprotease-amylase. His family history was significant for diabetes and hypertension.

On physical examination, his vital signs were stable, and he was afebrile. Cardiac, respiratory, abdominal, and right lower extremity examination was unremarkable. By contrast, extension of his left lower extremity was limited to only 20 degrees. No erythema, swelling, or deformity of the affected limb was present. The posterior thigh and calf were mildly tender to palpation. Pulses were positive bilaterally in the extremities.

Initial laboratory evaluation showed several out of range values including sodium $134 \mathrm{mmol} / \mathrm{L}$ (range $136-145 \mathrm{mEq} / \mathrm{L}$ ), blood urea nitrogen $29 \mathrm{mg} / \mathrm{dL}$ (range $5-20 \mathrm{mg} / \mathrm{dL}$ ), creatinine $1.7 \mathrm{mg} / \mathrm{dL}$ (range 0.6-1.2 mg/dL), random glucose $305 \mathrm{mg} / \mathrm{dL}$ (range $<200 \mathrm{mg} / \mathrm{dl}$ ), hemoglobin $12 \mathrm{~g} / \mathrm{dL}$ (range $14-17 \mathrm{~g} / \mathrm{dL}$ ), segmented neutrophil 86\%, C-reactive protein [CRP] $8.6 \mathrm{mg} /$ $\mathrm{dL}$ (range $0-1.0 \mathrm{mg} / \mathrm{dL}$ ), erythrocyte sedimentation rate 66 $\mathrm{mm} / \mathrm{hr}$ (range 0-17 mm/hr). Hemoglobin A1C (HbA1C) tested 3 months prior had been $10.3 \%$ (range $4-6 \%$ ). The creatinine kinase (CK) levels were normal at 67 (range 50-235 U/L). Other parameters were within normal range.

Doppler ultrasound of the left lower extremity ruled out deep venous thrombosis, and magnetic resonance imaging (MRI) revealed extensive edema with inflammatory changes involving several muscles in the thigh and calf on T1weighted images. Significant intramuscular inflammation and edema were present, particularly in the left thigh, with some superficial edema and enhancement also seen along the fascia.

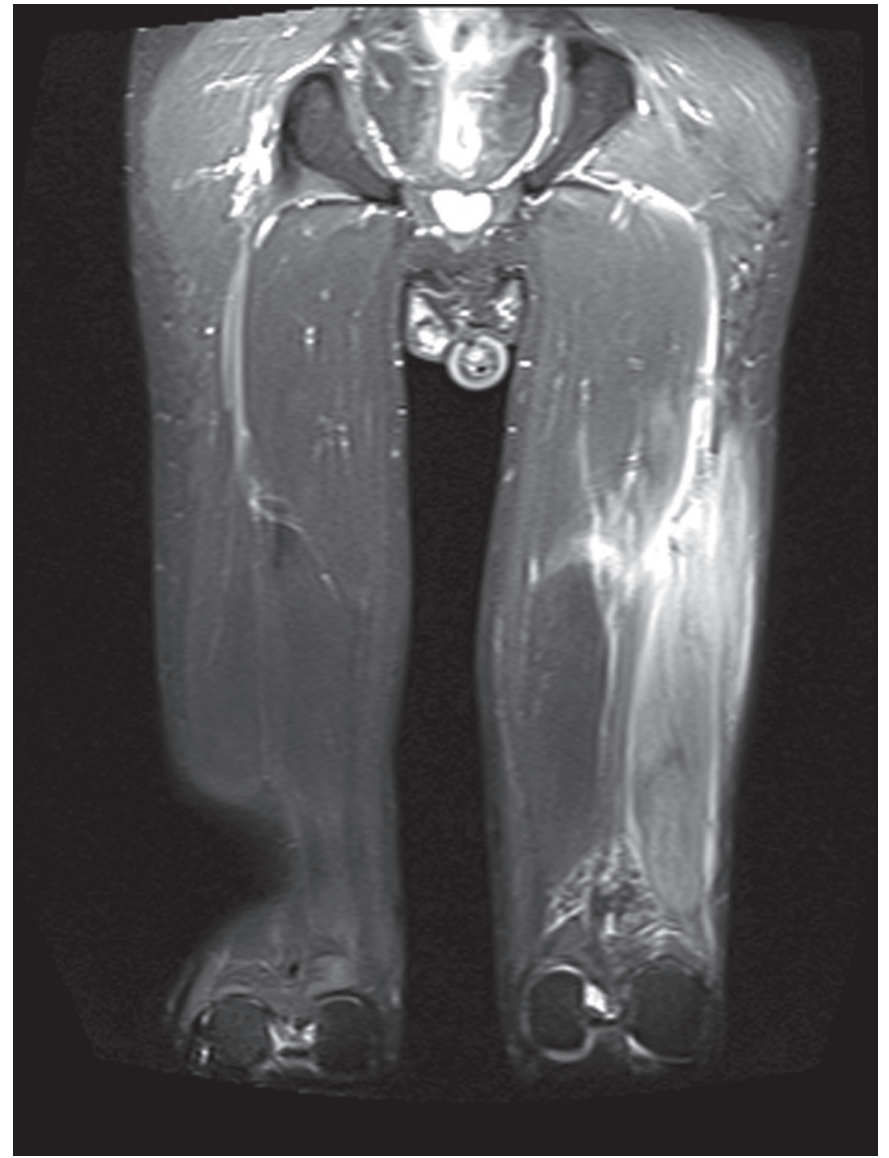

Figure 1: Fluid sensitive magnetic resonance coronal image of bilateral lower extremities taken posteriorly at the thighs. Image shows extensive left biceps femoris edema. There was an area of nonenhancement of the left distal biceps femoris muscle, suspicious for muscle infarction.

Areas of inflammation were also seen along the deep fascia of the left posterolateral distal thigh. There was an area of nonenhancement of the left distal biceps femoris muscle which indicated muscle infarction (figure 1). Differentiation between presence of infection versus inflammation associated with muscle necrosis was distinguished by identifying elevated levels of inflammatory markers (erythrocyte sedimentation rate [ESR] and CRP), normal white cell count, and a normal CK (characteristically seen in diabetic myonecrosis despite the breakdown of muscle). Prior history of diabetes and chronic immunosuppression with steroids advanced diabetic myonecrosis as the most likely diagnosis, since the patient appeared nontoxic with a low index of suspicion for an infectious etiology.

Although biopsy is the gold standard for a definitive diagnosis of diabetic myonecrosis, no biopsy was performed on the patient because it was not in the clinical best interest of the patient for the following reasons. The patient described in this case report had undergone a bilateral lung transplant consequential to underlying CF. Because of his transplantation status, he was on chronic immunosuppressant medication. Furthermore, with an $\mathrm{HbA1C}$ of $10.3 \%$, his risk for poor 
wound healing and infection were increased. The increased risks for complications, including impaired wound-healing and hemorrhage, ${ }^{6}$ prompted a cautionary approach to consideration of undertaking muscle biopsy. While lack of a confirmatory biopsy is a limitation of this report, the MRI findings were sufficiently robust to support the diagnosis.

Conservative management was initiated, with treatment including complete bed rest, analgesics, an antiplatelet agent, aspirin $81 \mathrm{mg} /$ day, and adequate glycemic control. Physical therapy was specifically avoided. The patient reported significant improvement in symptoms within the 3 -day period of hospitalization. He was off crutches within a week and completely pain-free by 4 -weeks post-discharge.

\section{Discussion}

In patients with CF, CFRD is a common comorbidity with prevalence as high as $50 \%$ in adult patients, increasing with age. ${ }^{2}$ Combined characteristics of both type 1 and type 2 diabetes are exhibited in CFRD, and it is primarily caused by underlying insulin insufficiency, although insulin resistance related to acute or chronic illness may play a role. ${ }^{2}$

First described by Angervall and Stener in 1965, ${ }^{7}$ diabetic myonecrosis is an uncommon complication of poorly controlled DM. Since then, more than a hundred such cases have been reported. Trujillo-Santos, ${ }^{8}$ in a systematic review of all reported cases of diabetic myonecrosis, found that it was more common in women (61.5\% of all cases), in type 1 diabetes $(59 \%$ of all cases), and in long-standing diabetes (mean duration of disease 14.3 years). Typical clinical presentation of diabetic myonecrosis consists of sudden onset of pain in the affected muscle associated with swelling and most commonly affecting thigh muscles followed by calf muscles. ${ }^{3}$ In his survey of 115 cases of diabetic myonecrosis in 2003, Trujillo-Santos ${ }^{8}$ reported that bilateral involvement was reported in only 14 cases ( $8.4 \%$ of the total surveyed). This would suggest that bilateral presentation is rarer, and that the presentation of unilateral symptoms in the index patient was consistent with the more common form of presentation of diabetic myonecrosis. Rarer case reports have cited involvement of paralumbar spine muscle ${ }^{9}$ of the legs and the neck muscles. ${ }^{10}$

Our patient reported improvement in symptoms in a few days and complete resolution in a month. Trujillo-Santos ${ }^{8}$ cited 2 to 17 weeks for resolution, with 4 weeks being average. Improvement with bed rest and steroids can be seen in days. Follow-up visits at 1 week and 4 weeks indicated steady progress with complete resolution of symptoms (pain), and need for ambulatory assist aids within 4 weeks.

While the pathogenesis of diabetic myonecrosis remains unclear, atheroembolism of the small vessels has been proposed. ${ }^{11}$ However, since the genetic defect in CF leads to malabsorption of fats, CF patients generally exhibit low total and low-density lipoprotein (LDL) cholesterol. Given the low cholesterol levels in our patient, atheroembolism of the small vessels as a pathogenic mechanism seems unlikely. Furthermore, despite low total cholesterol and LDL levels, the patient had developed microvascular disease (diabetic nephropathy and peripheral neuropathy), suggesting that microvascular disease may not be as dependent upon atherosclerosis in this group of patients. An alternative mechanism proposed for diabetic myonecrosis includes underlying abnormalities in the clotting cascade or fibrinolytic pathway, although this has not been well elucidated. The fact that $\mathrm{CF}$ patients can develop diabetic myonecrosis while presenting with a history of low cholesterol levels suggests that further research is warranted in elucidating the mechanism(s) underlying this complication of diabetes. Furthermore, the patient exhibited high levels of inflammatory markers (CRP was 8.6 and ESR 66). This pro-inflammatory state, along with his unusually poor diabetic control ( $\mathrm{HbA1C}$ of $10.3 \%$ ) may have played a role in the pathogenesis of diabetic myonecrosis in this patient.

In our patient, poor control of his diabetes was likely exacerbated by continuation of steroidal treatment necessitated by the lung transplantation in 2008. In this context, diabetic myonecrosis has been reported in patients with orthotopic heart transplant ${ }^{12}$ and islet cell transplant ${ }^{10}$ and was attributed to emergent diabetes which developed consequential to immunosuppressive treatment. ${ }^{13}$ It is not certain how common diabetic myonecrosis is in transplanted patients. In our review of the literature using the MeSH term 'diabetic myonecrosis' we found only 2 reports ${ }^{10,12}$ of diabetic myonecrosis occurring in transplant patients. Potentially, patients with $\mathrm{CF}$ are at increased risk for diabetic myonecrosis due to underlying CF-related diabetes mellitus coupled with post-transplant steroid-induced hyperglycemia.

Exhibiting features of both type 1 and type 2 diabetes, CFRD is associated with decreased insulin production and increased insulin resistance. Although the primary contributing defect is decreased production of insulin due to destruction of beta cells, emerging insulin resistance is initially the main stimulus of CFRD, secondary to acute infections. Affected patients exhibit decreased peripheral glucose uptake and poor insulin suppression of hepatic glucose production which concomitantly increases insulin resistance. ${ }^{14}$ Patients with CFRD have poorly controlled diabetes in general because of malabsorption and consequential high caloric needs associated with an underlying deficiency in pancreatic enzymes, leading to abnormalities in bile salts, mucosal transport problems, and variance in motility.

The imaging modality of choice for soft tissue evaluation is MRI, which shows findings of increased signal on T2 imaging in patients presenting with edema and no discrete masses. Although tissue biopsy can be used to confirm the diagnosis, it is not required for diagnosis, since MRI and a thorough clinical evaluation are generally sufficient. ${ }^{7}$ In the case of our patient, who was being treated with immunosuppressive 
medication, biopsy was not pursued since the diagnosis was well supported by MRI findings, and the risk of performing a biopsy outweighed its benefits. $\mathrm{CK}$ levels in our patient were normal (67 U/L [normal range: 50-235 U/L]). In their survey of 115 diabetic myonecrosis cases in 2003, TrujilloSantos $^{7}$ similarly reported normal CK levels in $52.7 \%$ of the cases. Differential diagnoses of diabetic myonecrosis should include abscess, cellulitis, mass, hemorrhage, polymyositis, dermatomyositis, and deep vein thrombosis.

Diabetic myonecrosis is normally self-limited, and good glycemic control with supportive care is the mainstay of treatment. Analgesia and immobilization can aid in decreasing pain. Exercise or physical therapy should be expressly avoided, as it may worsen pain and myonecrosis. The index patient responded well to these therapeutic approaches.

\section{Conclusion}

To our knowledge, this is the first published case of a CFRD patient presenting with diabetic myonecrosis. The only other report found was a very recent abstract of a case presented by Kopp et $\mathrm{al}^{15}$ at the American Thoracic Society International Conference in May 2012. A high index of suspicion for diabetic myonecrosis is warranted when a patient with CFRD presents with lower extremity pain.

\section{Acknowledgements}

The authors acknowledge the Marshfield Clinic Research Foundation's Office of Scientific Writing and Publication for assistance in preparing this manuscript.

\section{References}

1. Lobo J, Rojas-Balcazar JM, Noone PG. Recent advances in cystic fibrosis. Clin Chest Med 2012;33:307-328.

2. Marshall BC, Butler SM, Stoddard M, Moran AM, Liou TG, Morgan WJ. Epidemiology of cystic fibrosis-related diabetes. J Pediatr 2005;146:681-687.

3. Choudhury BK, Saikia UK, Sarma D, Saikia M, Choudhury SD, Bhuyan D. Diabetic myonecrosis: An underreported complication of diabetes mellitus. Indian J Endocrinol Metab 2011;15:S58-S61.

4. Cystic Fibrosis Foundation Patient Registry. 2011 Annual Data Report. Bethesda, Maryland. (C)2012 Cystic Fibrosis Foundation. Available at: http://www.cff.org/UploadedFiles/ research/ClinicalResearch/2011-Patient-Registry.pdf. Last accessed: August 22, 2013.

5. Johnson S, Knox AJ. Arthropathy in cystic fibrosis. Respir Med 1994;88:567-570.

6. Keller DR, Erpelding M, Grist T. Diabetic muscular infarction. Preventing morbidity by avoiding excisional biopsy. Arch Intern Med 1997;157:1611-1612.

7. Angervall L, Stener B. Tumoriform focal muscle degeneration in two diabetic patients. Diabetologia 1965;1:39-42.

8. Trujillo-Santos AJ. Diabetic muscle infarction: an underdiagnosed complication of long-standing diabetes. Diabetes Care 2003;26:211-215.

9. Kim SW, Kim SS. Myonecrosis of paralumbar spine muscle. Spine (Phila Pa 1976) 2011;36:E1162-E1165.

10. Salehi P, Stull MA, Martellotto J, Gangemi A, Hatipoglu B, Benedetti E, Oberholzer J. Case report: diabetic myonecrosis of the neck complicated by infection in an islet transplanted patient. J Diabetes Complications 2009;23:140-142.
11. Hoyt JR, Wittich CM. Diabetic myonecrosis. J Clin Endocrinol Metab 2008;93:3690.

12. Byars JA, Rosenberg EI. Diabetic myonecrosis in an orthotopic heart transplant recipient. J Heart Lung Transplant 2007;26:549-552.

13. Marchetti P. New-onset diabetes after transplantation. J Heart Lung Transplant 2004;23:S194-S201.

14. Moran A, Becker D, Casella SJ, Gottlieb PA, Kirkman MS, Marshall BC, Slovis B; CFRD Consensus Conference Committee. Epidemiology, pathophysiology, and prognostic implications of cystic fibrosis-related diabetes: a technical review. Diabetes Care 2010;33:2677-2783.

15. Kopp BT, McCoy K, Kirkby S. Sugar Pains: Novel Diabetic Myonecrosis in a Cystic Fibrosis Patient. Am J Respir Crit Care Med 2012;185:A3382. [Abstract]

\section{Author Affiliations}

Sreelatha Chalasani, MD*; Shankar S. Bettadahalli, MD*; Satya V. Bhupathi, MD*; and Vijay H. Aswani, MD, PhD

${ }^{*}$ Department of Internal/Hospital Medicine, Marshfield

Clinic, Marshfield, Wisconsin, USA

'Department of Endocrinology, Marshfield Clinic, Marshfield, Wisconsin, USA

*Department of Internal Medicine \& Pediatrics (Med/Peds), Marshfield Clinic, Marshfield, Wisconsin, USA 